\title{
Uso de Simuladores como Estrategia de Aprendizaje de Habilidades Técnicas en Enfermería de la UNAMAD
}

Use of Simulators as a Learning Strategy for Technical Skills in Nursing at UNAMAD

Uso de Simuladores como Estratégia de Aprendizagem de Habilidades Técnicas em Enfermagem na UNAMAD

\section{ARTÍCULO GENERAL}

\author{
Nola Dacia Espejo Landeo \\ https://orcid.org/0000-0001-9594-1708 \\ nespejo@unamad.edu.pe
}

Universidad Nacional Amazónica de Madre de Dios, Madre de Dios - Perú

Recibido 15 de Febrero 2021 | Arbitrado y aceptado 03 de Marzo 2021 | Publicado el 01 de Junio 2021

\section{RESUMEN}

La presente investigación: Uso de Simuladores como Estrategia de Aprendizaje de Habilidades Técnicas en la Facultad de Enfermería de la Universidad Nacional Amazónica de Madre de Dios, tiene como objetivo principal determinar la influencia del Uso de Simuladores como Estrategia de Aprendizaje de Habilidades Técnicas en la Facultad de Enfermería de la Universidad Nacional Amazónica de Madre de Dios. El tipo de investigación es descriptiva, explicativa, cuantitativa. La población es de 271 individuos, constituido por: 250 estudiantes y 21 docentes. Se elaboraron dos instrumentos tipo cuestionario una para cada variable, se sometieron a confiabilidad con el estadístico demostrando consistencia interna del instrumento con el valor Coeficiente $\alpha$ Cronbach $=0,831$ y 0.876 respectivamente lo que nos indica un índice de consistencia "bueno". Finalmente, la investigación concluye en que se demuestra que el "Uso de simuladores" como estrategia de Aprendizaje, influye significativamente en el desarrollo de las "Habilidades Técnicas" en la Facultad de Enfermería de la Universidad Nacional Amazónica de Madre de Dios.

Palabras clave: Uso de Simuladores; Estrategia de Aprendizaje

\section{ABSTRACT}

The present investigation: Use of Simulators as a Learning Strategy for Technical Skills in the Faculty of Nursing of the National Amazon University of Madre de Dios, has as its main objective to determine the influence of the Use of Simulators as a Learning Strategy for Technical Skills in the Faculty of Nursing from the National Amazon University of Madre de Dios. The type of research is descriptive, explanatory, quantitative. The population is 271 individuals, consisting of: 250 students and 21 teachers. Two questionnaire-type instruments were prepared, one for each variable, they were subjected to reliability with the statistic demonstrating internal consistency of the instrument with the Cronbach $\alpha$ Coefficient value $=0.831$ and 0.876 respectively, which indicates a "good" consistency index. Finally, the research concludes that it is shown that the "Use of simulators" as a learning strategy significantly influences the development of "Technical Skills" in the Faculty of Nursing of the National Amazon University of Madre de Dios.

Keywords: Use of Simulators; Learning strategy

\section{RESUMO}

A presente investigação: Uso de Simuladores como Estratégia de Aprendizagem de Habilidades Técnicas na Faculdade de Enfermagem da Universidade Nacional da Amazônia de Madre de Dios, tem como objetivo principal determinar a influência do Uso de Simuladores como Estratégia de Aprendizagem de Habilidades Técnicas na Faculdade de Enfermagem da Universidade Nacional da Amazônia de Madre de Dios. O tipo de pesquisa é descritiva, explicativa, quantitativa. A população é de 271 indivíduos, sendo: 250 alunos e 21 professores. Foram elaborados dois instrumentos do tipo questionário, um para cada variável, os quais foram submetidos à confiabilidade com a estatística demonstrando consistência interna do instrumento com valor do Coeficiente $\alpha$ de Cronbach $=0,831$ e 0,876 respectivamente, o que indica um índice de consistência "bom". Por fim, a pesquisa conclui que é demonstrado que o "Uso de simuladores" como estratégia de aprendizagem influencia significativamente o desenvolvimento de "Habilidades Técnicas" na Faculdade de Enfermagem da Universidade Nacional da Amazônia de Madre de Dios.

Palavras-chave: Uso de Simuladores; Estratégia de aprendizagem 


\section{Introducción}

La educación en los últimos tiempos experimenta cambios basados generalmente en la manera de innovar para evitar que las sesiones de clases sean meros tradicionalistas, somos conscientes que los estudiantes de hoy deben ser los propios propulsores de su aprendizaje, el docente por su parte debe promover la metodología activa en todo momento, siendo esta parte una visión multidisciplinaria; por ello, las tendencias de la educación en el área de salud específicamente en enfermería, requieren mucho de dichos cambios para promover en el estudiante el interés y la satisfacción en su formación profesional con criterio de integralidad. Ciertamente, se exige entre otros elementos de la enseñanza en enfermería, incorporar el uso de simuladores a aplicar la simulación durante las clases demostrativas prácticas en los laboratorios de ensayo.

El método de usar simuladores en la enseñanza desde sus inicios ha mostrado ser una estrategia muy pertinente que permite que los docentes y estudiantes interactúen de la manera más próxima a lo real, de esta forma el estudiante gana experticia en el campo de acción, será un experto y decidido al momento de proceder en la realidad ante pacientes de diferente etiología de enfermedad o padecimiento.

Según, Amaro, et.al (2019), "a principios del siglo XX, los estudiantes de enfermería tenían las denominadas salas de arte, que eran lugares destinados para realizar prácticas y desarrollar algunos procedimientos con el fin de mejorar la atención del paciente". Desde entonces, los simuladores se van perfeccionando cada vez mejor, con la característica de aproximarse más a la realidad.

Para, Enríquez y Perretta (2014) así también, Díaz et.al (2014) respectivamente, “la simulación ha resultado ser una herramienta educativa con una metodología innovadora basada en un modelo de enseñanza constructivista, donde el conocimiento no se descubre, se construye". Por consiguiente, los estudiantes participan de forma activa durante todo su proceso de aprendizaje, ganando experiencia en el desarrollo sus habilidades, para accionar con rapidez, seguridad y mesura ante una práctica cuya característica es muy semejante a la situación realística, contribuyendo propositivamente en la adquisición de diversos conocimientos, habilidades destrezas, es decir capacidades para accionar como personal de enfermería competente. 
El uso de simuladores en la enseñanza a través de los años ha permitido ganar experiencia también en la enseñanza, es así que, Martínez y Matus (2015), "utilizaron casos clínicos simulados, en donde los estudiantes realizaban el rol de enfermería a cargo del paciente y debían tomar las decisiones para realizar las intervenciones más adecuadas". Por su parte, Velasco (2013), utilizó en su estudio “todas las fases didácticas; la sesión informativa previa, introducción, reunión sobre el manejo del simulador, teoría, reunión o discusión sobre el caso, escenario, debriefing y conclusión”.

Gracias a los beneficios que permite alcanzar, el uso de simuladores en el desarrollo de las habilidades técnicas en enfermería, incluso la Organización Mundial de la Salud (OMS), apoya para que la estrategia de usar simuladores se masifique en todas las universidades en las cuales existan áreas de formación en salud, con el propósito de fortalecer y mejorar la calidad de la enseñanza y el aprendizaje de los estudiantes de enfermería. Es fundamental que los estudiantes adquieran conocimientos necesarios para desarrollar su práctica clínica, haciendo uso de simuladores.

Por esta razón, es que decidimos el estudio para demostrar la importancia de esta herramienta en el aprendizaje, con el objetivo de Determinar la influencia del Uso de Simuladores como Estrategia de Aprendizaje de Habilidades Técnicas en la Facultad de Enfermería de la Universidad Nacional Amazónica de Madre de Dios.

\section{Materiales y métodos}

La investigación es: descriptiva, explicativa, cuantitativa.

Descriptiva, considerado así en razón a que se teoriza las características de la población materia en estudio.

Explicativa, porque la investigación explora la situación causal respecto a las variables determinadas para el estudio, no solo describen o acercan a la situación de la problemática de investigación, sino también, trata de ubicar las causas del mismo.

Cuantitativa, debido a que hace uso de la estadística para los análisis de datos correspondientes, para brindar respuesta a las interrogantes de la investigación y de esta manera corroborar las hipótesis correspondientes planteadas. 
La Población está conformada por total de 271 individuos, constituido por: 250 estudiantes y 21 docentes de la Facultad de Enfermería de la Universidad Nacional Amazónica de Madre de Dios. (UNAMAD).

La muestra se determinó de manera no aleatoria, con intencionalidad, con conocimiento de causa de forma aleatoria simple.

Las técnicas utilizadas para obtener información sobre las variables de la investigación fueron a través de los Análisis documentales de la misma facultad y la Encuesta aplicada a los estudiantes y docentes, asimismo, el instrumento utilizado para la recopilación de datos de acuerdo a las variables son dos encuestas tipo cuestionario: uno para cada variable.

Se realizaron los siguientes procedimientos sucesivos: La confiabilidad estadística del instrumento "uso de simuladores" y "habilidades técnicas" se determinó mediante el estadístico de prueba Alfa de Cronbach, con un valor Coeficiente $\alpha=0,831$, y 0,876 respectivamente.

Estadística descriptiva: Análisis descriptivo de las variables implicadas.

Distribución de frecuencias mediante los cuadros y figuras estadísticas.

Estadística inferencial: Dado que las variables son de tipo categóricas y escala ordinal, realizamos la correlación con el estadístico: "Rho de Spearman".

Posteriormente se realizó la discusión de los resultados mediante la confrontación de los mismos con los antecedentes citados.

\section{Resultados}

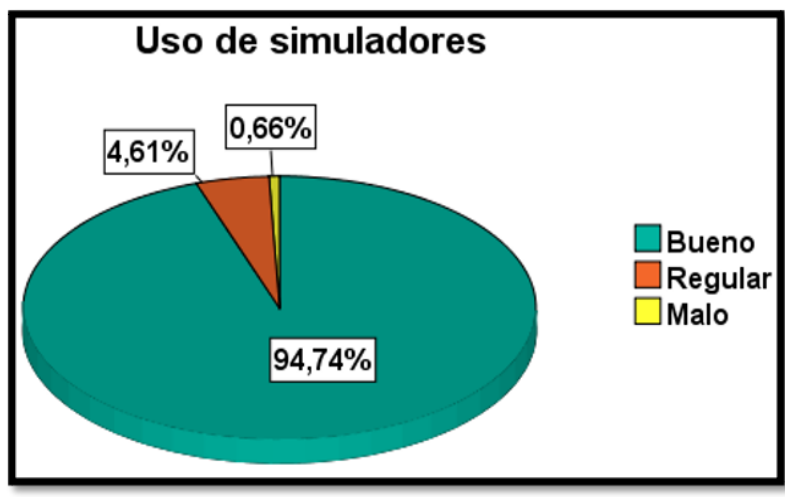

Fuente: Elaboración propia. 
En la presente gráfica se muestra el resumen de los resultados obtenidos para la variable "Uso de simuladores" dentro del estudio: "Uso de Simuladores como Estrategia de Aprendizaje de Habilidades Técnicas en la Facultad de Enfermería de la Universidad Nacional Amazónica de Madre de Dios". En esta se observa que dicho uso de simuladores es bueno para el $94,74 \%$ de los entrevistados, regular para el 4,61\%, y es malo solo para el $0,66 \%$ del total.

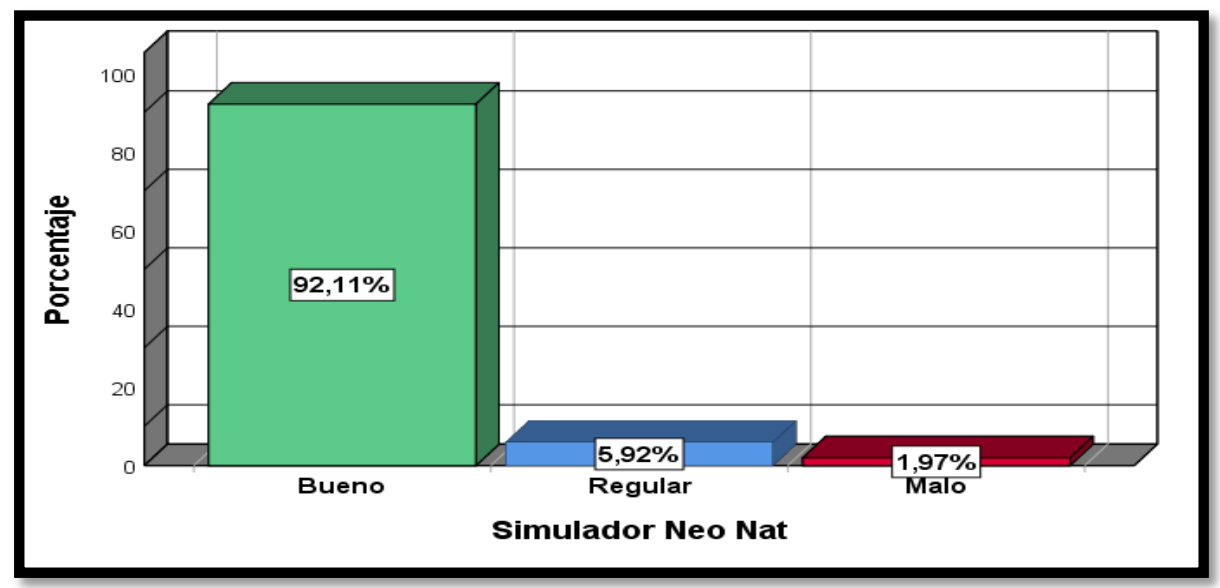

\section{Fuente: Elaboración propia.}

En la presente gráfica se muestra el resumen de los resultados obtenidos para la dimensión "Simulador Neo Nat" dentro del estudio: "Uso de Simuladores como Estrategia de Aprendizaje de Habilidades Técnicas en la Facultad de Enfermería de la Universidad Nacional Amazónica de Madre de Dios”. En esta se observa que dicho uso del simulador Neo Nat es bueno para el $92,11 \%$ de los entrevistados, regular para el $5,92 \%$, y es malo solo para el $1,97 \%$ del total. 


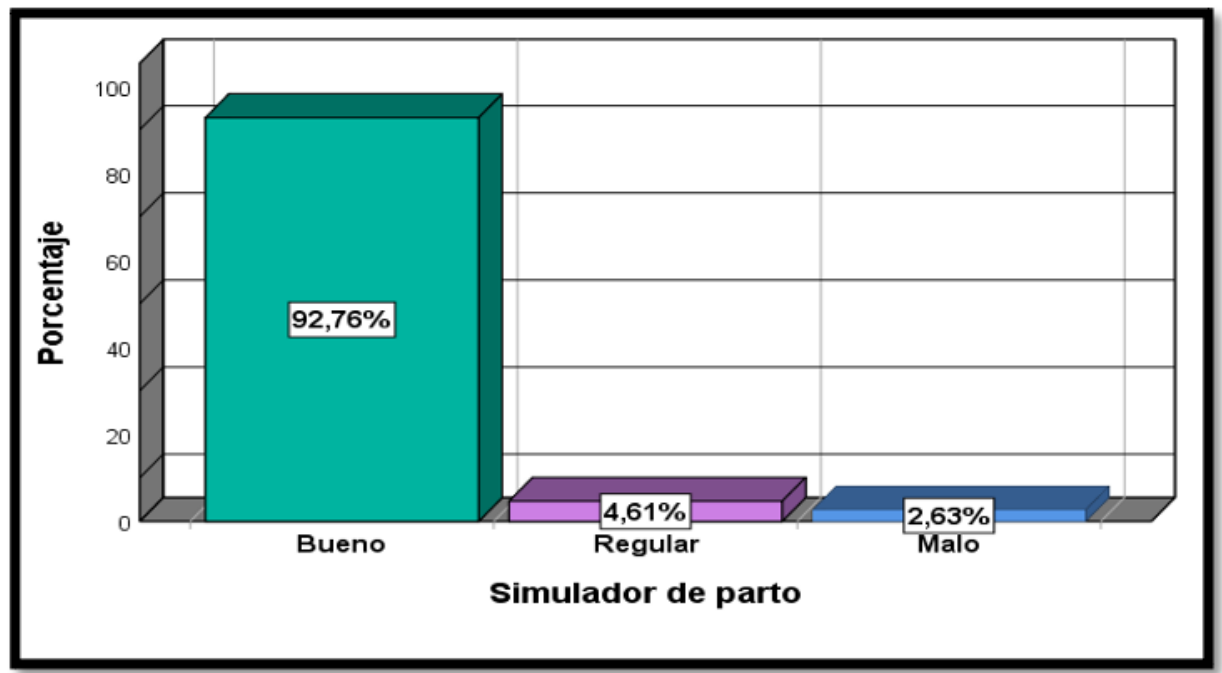

\section{Fuente: Elaboración propia.}

En la presente gráfica se muestra el resumen de los resultados obtenidos para la dimensión "Simulador de parto" dentro del estudio: "Uso de Simuladores como Estrategia de Aprendizaje de Habilidades Técnicas en la Facultad de Enfermería de la Universidad Nacional Amazónica de Madre de Dios”. En esta se observa que dicho uso del simulador de parto es bueno para el $92,76 \%$ de los entrevistados, regular para el $4,61 \%$, y es malo solo para el $2,63 \%$ del total.

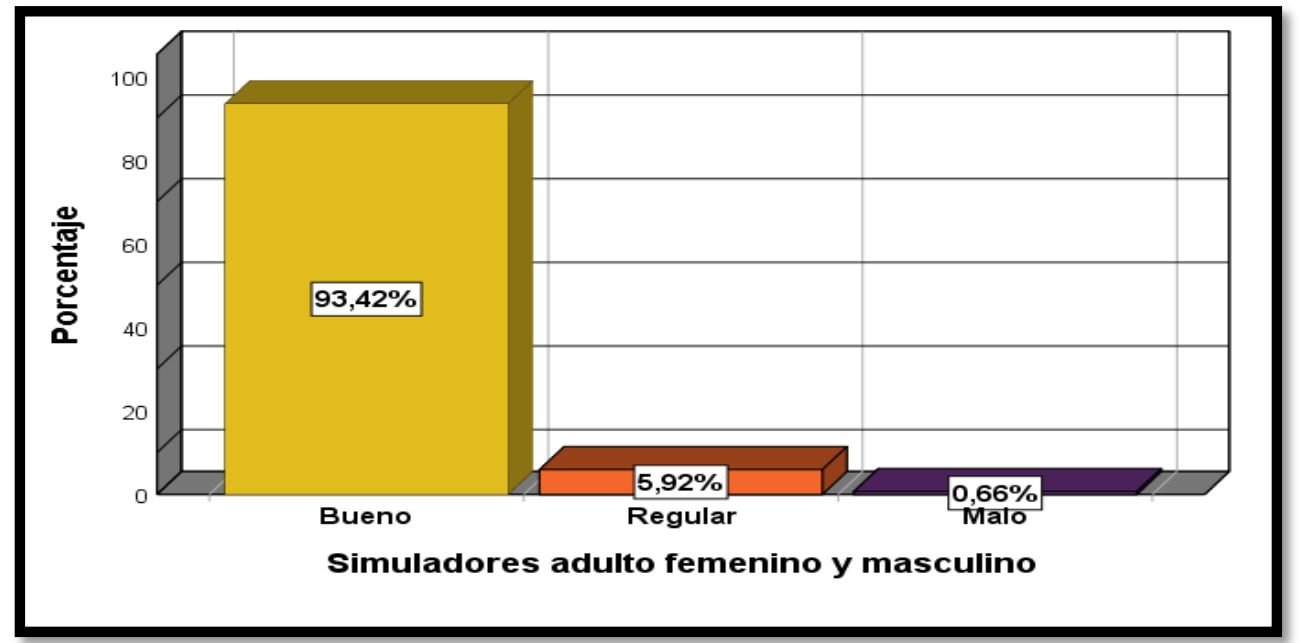

Fuente: Elaboración propia. 
En la presente gráfica se muestra el resumen de los resultados obtenidos para la dimensión "Simulador adulto femenino y masculino" dentro del estudio: "Uso de Simuladores como Estrategia de Aprendizaje de Habilidades Técnicas en la Facultad de Enfermería de la Universidad Nacional Amazónica de Madre de Dios”. En esta se observa que dicho uso del simulador adulto femenino y masculino es bueno para el $93,42 \%$ de los entrevistados, regular para el 5,92\%, y es malo solo para el 0,66\% del total.

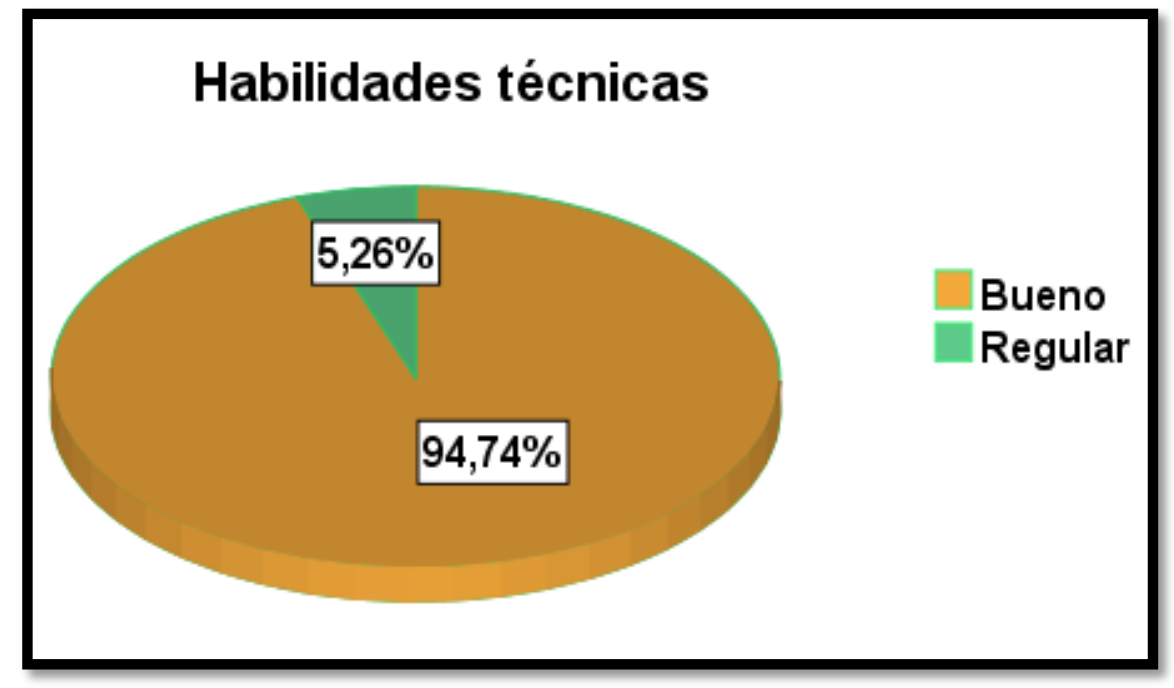

\section{Fuente: Elaboración propia}

En la presente gráfica se muestra el resumen de los resultados obtenidos para la variable "Habilidades técnicas" dentro del estudio: "Uso de Simuladores como Estrategia de Aprendizaje de Habilidades Técnicas en la Facultad de Enfermería de la Universidad Nacional Amazónica de Madre de Dios”. En esta se observa que dichas habilidades técnicas son buenas para el $94,74 \%$ de los entrevistados, regular para el 5,26\%, y mala para ninguno.

\section{Contrastación de la hipótesis general}

El Uso de Simuladores como Estrategia de Aprendizaje influye significativamente en las Habilidades Técnicas en la Facultad de Enfermería de la Universidad Nacional Amazónica de Madre de Dios. 
Para comprobar esto se plantean las siguientes hipótesis de trabajo:

H1: El Uso de Simuladores como Estrategia de Aprendizaje influye significativamente en las Habilidades Técnicas en la Facultad de Enfermería de la Universidad Nacional Amazónica de Madre de Dios.

Las variables en cuestión no son independientes.

Ho: El Uso de Simuladores como Estrategia de Aprendizaje, no influye significativamente en las Habilidades Técnicas en la Facultad de Enfermería de la Universidad Nacional Amazónica de Madre de Dios.

\section{Nivel de significancia (nivel de riesgo)}

$\alpha=0,05(5 \%)$

\begin{tabular}{|c|c|c|c|c|}
\hline \multicolumn{5}{|c|}{ Correlaciones } \\
\hline & & & $\begin{array}{l}\text { Uso de } \\
\text { Simuladores }\end{array}$ & $\begin{array}{l}\text { Habilidades } \\
\text { Técnicas }\end{array}$ \\
\hline \multirow[t]{6}{*}{$\begin{array}{l}\text { Rho de } \\
\text { Spearman }\end{array}$} & \multirow{3}{*}{$\begin{array}{l}\text { Uso de } \\
\text { Simuladore } \\
\text { s }\end{array}$} & $\begin{array}{l}\text { Coeficiente de } \\
\text { correlación }\end{array}$ & 1,000 &, $877 * *$ \\
\hline & & Sig. (bilateral) & . & ,002 \\
\hline & & $\mathrm{N}$ & 19 & 19 \\
\hline & \multirow[t]{3}{*}{$\begin{array}{l}\text { Habilidades } \\
\text { Técnicas }\end{array}$} & $\begin{array}{l}\text { Coeficiente de } \\
\text { correlación }\end{array}$ & ,877** & 1,000 \\
\hline & & Sig. (bilateral) & ,002 & . \\
\hline & & $\mathrm{N}$ & 19 & 19 \\
\hline
\end{tabular}

\section{Coeficiente de correlación: 0,877}

Sig. (bilateral / p valor) : 0,002

\section{Decisión Estadística:}

Puesto que: $0,002<0,05$ se acepta la hipótesis del investigador $(\mathrm{H} 1)$ y se rechaza la hipótesis nula (H0). 


\section{CONCLUSIONES}

Con respecto a la hipótesis general: El Uso de Simuladores como Estrategia de Aprendizaje influye significativamente en las Habilidades Técnicas en la Facultad de Enfermería de la Universidad Nacional Amazónica de Madre de Dios.

- El valor obtenido Sig. $=0,002$ que se muestra en la tabla, nos permite afirmar con un nivel de confianza del 95\% que: SÍ existe asociación estadísticamente significativa entre el Uso de Simuladores como Estrategia de Aprendizaje y la adquisición de Habilidades Técnicas en la Facultad de Enfermería de la Universidad Nacional Amazónica de Madre de Dios.

- Las variables en cuestión no son independientes.

- Además se concluye que: existe correlación positiva fuerte entre las variables analizadas.

Se demuestra que el Uso de Simuladores como Estrategia de Aprendizaje influye significativamente en las Habilidades Técnicas en la Facultad de Enfermería de la Universidad Nacional Amazónica de Madre de Dios.

\section{REFERENCIAS BIBLIOGRÁFICAS}

Alessi, S. (2000). Simulation desing for training and assessment. En O'Neil, \& D. Andrews, Aircrew training and assessment (págs. 197-222). Mahwah, NJ: Erlbaum.

Amaro-López, L., Hernández-González, P., Hernández-Blas, A., y Hernández Arzola, L. (2019). La simulación clínica en la adquisición de conocimientos en estudiantes de la Licenciatura de Enfermería. Enfermería Universitaria, 16(4). doi: https://doi.org/10.22201/eneo.23958421e.2019.4.543

Arreciado-Marañón, A., \& Estorach-Querol, M. F.-F. (2011). La enfermera experta en el cuidado del paciente crítico según Patricia Benner. Enfermería Intensiva, 22(3):1126. 
Bandura, A. (1987). Teoría del Aprendizaje Social. Madrid: Espasa-Calpe.

Brill, R., Spevetz, A., \& Branson, R. (2001). Critical care delivey in the intensive care unit: defining clinical roles and the best practice model. Crit Care Med, 29:2007-19.

Carmona JV, Santonja L. (2016). Simulación clínica: del posgrado al grado. Enfermería integral. 112: 64-66.

Casal Angulo María del Carmen (2016). La simulación como metodología para el aprendizaje de habilidades no técnicas en Enfermería. Tesis doctoral. Programa de doctorado en enfermería. Facultad de Enfermería y Podología de la Universidad de Valencia. Valencia - España.

Chisari, G., Brown, C., Calkins, M., Echthernacht, M., Kearney-Nunnery, R., Knopp, B., \& Spector, N. (2005). Clinical instruction in prelicensure nursing program. National Council of State Boards of Nursing (NCSBN).

Clemente Lino, Y. Y., Contreras Dávila, R. T., \& Tucto Bustamante, L. M. (2016). Efectividad del uso de simuladores clínicos en el proceso enseñanza aprendizaje en los estudiantes de la asignatura Enfermería básica y aplicación de tecnologías I - Facultad de Enfermería - UNHEVAL-2016.

Enriquez, DS, Perretta, C. (2014). Enseñanza de la Pediatría con simuladores. IntraMed j. 3(2): 8 pantallas. https://bit.ly/2NxOZok

Fidias G. Arias (2012). El Proyecto de Investigación Introducción a la metodología científica $6^{\text {a }}$ Edición Editorial Episteme

Galindo, J., \& Visbal, L. (2007). Simulación, herramienta para la educación médica. Salud Uninorte, 23 (1):79-95.

González Gómez, J. (2008). Nuevas metodologías en el entrenamiento de emergencias pediátricas: simulación médica aplicada a pediatría. An Pediatr, 6:612-20.

Hernández, R., Fernández, C., Baptista, M. (2010), Metodología de la Investigación Científica, México: Mc Graw-Hill

Hoyos, P., Cardona, M., \& Correa, D. (2008). Humanizar los contextos de salud, cuestión de liderazgo. Invest. Educ. Enfer, 26 (2):218-225. 
Jeffries. (2003). Designing, implementing and evaluating simulations used as teaching strategies in nursing. Nursing Education Perspectives, 26 (2), 96-103.

López, M., Ramos, L., Pato, O., \& López, S. (2013). La Simulación clínica como herramienta de aprendizaje. Cirugía Mayor ambulatoria, 18 (1) 25 - 29.

Martínez F, Matus R. (2015). Desarrollo de habilidades con simulación clínica de alta fidelidad. Perspectiva de los estudiantes de enfermería. Enferm. univ. 12 (2): 93-8 http://dx.doi.org/10.1016/j.reu.2015.04.003

Miller, K., Riley, W., \& Davis, S. (2009). Identifying key nursing and team behaviours to achieve high reliability. Journal of Nursing Management, 17,247-255.

Riquelme Hernández, G., Acevedo Martínez, V., \& Muñoz Guerrero, X. (2017). Incorporación de la simulación en la enseñanza de los contenidos de parto y atención al recién nacido en Enfermería. Educación Médica Superior, 31(4). Recuperado de http://www.ems.sld.cu/index.php/ems/article/view/1113/585

Rubio, R. (2012). Pasado, presente y futuro de la simulación en Anestesiología. Revista Mexicana de Anestesiología, 35 (3)186-191.

Saaranen, T., Vaajoki, A., Kellomäki, M., \& Hyvärinen, M. (2015). The simulation method in learning interpersonal communication competence-Experiences of masters'degree students of health sciences. Nurse Education Today, 35 e8-e13.

Salas, E., Rosen, M., \& King, H. (2007). Managing teams managing crises: principles for teamwork in the ER and beyond. Theorical Issues Ergonomics Science, 381-94.

Sherer, M., \& Eadie, R. (1987). Employability Skills: Key to Success. Thrust, 17 (2), $16-17$.

Tamayo y Tamayo, m. (2007). El proceso de la investigación científica; incluye glosario y manual de evaluación de proyectos (4a. Ed.). Guadalajara: Limusa.

Vargas, F. (2005). Key Competencies and Lifelong Learning. Montevideo: Cinterfor.

Velasco-Martín A. (2013). Simulación Clínica y Enfermería, creando un ambiente de simulación. [Trabajo de Grado]. Universidad de Cantabria. https://bit.ly/320utC1 
Vásquez Vásquez, María Gabriela (2019). Simuladores como metodología de aprendizaje: perspectiva del estudiante de enfermería de la universidad señor de Sipán, pimentel-2017. Tesis para optar el Título profesional de Licenciado en enfermería. Facultad de Ciencias de la Salud. Escuela Académico Profesional de Enfermería. Universidad Señor de Sipán. Línea de Investigación: Educación en Salud. Pimentel Perú. 